\section{Effect of Bias Current on Complexity and Time Delay Signature of Chaos in Semiconductor Laser with Time-Delayed Optical Feedback}

Ji, Songkun; Hong, Yanhua
\end{abstract}

\title{
IEEE Journal of Selected Topics in Quantum Electronics
}

\author{
DOI: \\ 10.1109/JSTQE.2017.2689328
}

Published: 01/11/2017

Peer reviewed version

Cyswllt i'r cyhoeddiad / Link to publication

Dyfyniad o'r fersiwn a gyhoeddwyd / Citation for published version (APA):

Ji, S., \& Hong, Y. (2017). Effect of Bias Current on Complexity and Time Delay Signature of Chaos in Semiconductor Laser with Time-Delayed Optical Feedback. IEEE Journal of Selected Topics in Quantum Electronics, 23(6), [1800706]. https://doi.org/10.1109/JSTQE.2017.2689328

\footnotetext{
Hawliau Cyffredinol / General rights

Copyright and moral rights for the publications made accessible in the public portal are retained by the authors and/or other copyright owners and it is a condition of accessing publications that users recognise and abide by the legal requirements associated with these rights.

- Users may download and print one copy of any publication from the public portal for the purpose of private study or research.

- You may not further distribute the material or use it for any profit-making activity or commercial gain

- You may freely distribute the URL identifying the publication in the public portal?
}

Take down policy

If you believe that this document breaches copyright please contact us providing details, and we will remove access to the work immediately and investigate your claim. 


\title{
Effect of Bias Current on Complexity and Time Delay Signature of Chaos in Semiconductor Laser with Time-Delayed Optical Feedback
}

\author{
Song-Kun Ji, Yanhua Hong, Member, IEEE
}

\begin{abstract}
The effect of bias current on the complexity and time-delay signature of chaotic signals in semiconductor lasers with polarization preserved optical feedback has been studied experimentally and theoretically. The peak value of the autocorrelation coefficient and the normalized permutation entropy at the feedback round trip time are used to quantify the time delay signature and complexity, respectively. The results show that the time-delay signature is approximately in an inverse relationship with the complexity of chaos when the semiconductor laser is subject to low or strong optical feedback. However, the inverse relationship disappears when the laser operates at higher bias currents with intermediate feedback strength. The simulation results are qualitatively agreed with the experimental results.
\end{abstract}

Index Terms - Chaos, optical feedback, complexity, time delay signature, permutation entropy, semiconductor lasers.

\section{INTRODUCTION}

C HAOS generated in semiconductor lasers with optical feedback has attracted considerable research interest due to its many potential applications, such as high-speed communications [1]-[7], chaotic logic gates [8], chaotic optical time-domain reflectors [9]-[11], chaotic lidars [12] and physical random number generators [13], [14]. The complexity, bandwidth and time delay (TD) signature of chaos are the three main parameters for assessing the suitability of its applications. The bandwidth and TD signature of chaos have been studied extensively [15]-[28]. Complexity of chaos has also been investigated [29]-[32]. Effect of optical injection strength, frequency detuning and feedback strength on the complexity, bandwidth and TD have been broadly reported. Temperature and bias current are the two primary parameters in the driving laser diodes. Examination of the effect of bias current on bandwidth, TD signature and complexity of chaos have been reported in some papers [22],[29], [31], [33], [34]. Oliver et al. [33] shows the details of the relationship between the TD signature and the bias current. Kanno et al. [31] have also numerically simulated complexity of chaos as a function of the

Manuscript received January 31, 2017. This work was supported in part by the Sêr Cymru National Research Network in Advanced Engineering and Materials and by International cooperation project of key research and development plan of Shanxi Province, China (Grant No. 201603D421008).

S. K. Ji and Y. H. Hong are with School of Electronic Engineering, Bangor University, Gwynedd LL57 1UT, Wales, UK. (e-mail: eep40a@bangor.ac.uk; y.hong@bangor.ac.uk). bias current. However, to the best of our knowledge, the influence of bias current on the relationship between complexity and TD signature has not been experimentally investigated in detail. In this paper, we experimentally study the effect of bias current on the complexity and TD signature with the different feedback strengths in semiconductor lasers subject to polarization preserved optical feedback. The relationship between the complexity and TD signature has also been investigated experimentally and theoretically. The difference between our experimental results and the simulation results [31] on the relationship between complexity and TD signature has been investigated using the Lang Kobayashi laser equations [35].

The remainder of this paper is organized as follows. The experimental setups and the operation parameters are described in Section II, followed by the analysis methods of TD signature and complexity of chaos in Section III. The experimental results are in section IV. The theoretical model and results are presented in section V. Finally, in section VI, conclusions are drawn based on the results obtained.

\section{EXPERIMENTAL SETUP}

Two experimental setups are used in this investigation. The first experiment was carried out in a free-space setup, as shown in Fig. 1(a). A single mode Fabry-Perot laser (APL 860-40) with lasing wavelength around $860 \mathrm{~nm}$ was used in the experiment. The laser was driven by an ultra-low noise current source and its temperature was controlled to an accuracy of 0.01 Celsius. At the operating temperature, the threshold current is $37.3 \mathrm{~mA}$. The laser was subject to the optical feedback from mirror M1. The feedback power was controllable with a natural density filter (ND1). The feedback round trip time was about 5.6ns. An optical isolator (ISO) was used to prevent any unwanted feedback from the detection path. The output of the laser was detected by $12 \mathrm{GHz}$ photodetectors and recorded by a $4 \mathrm{GHz}$ digital oscilloscope and a $30 \mathrm{GHz}$ bandwidth RF spectrum analyzer. The sampling rate of the oscilloscope was set at $10 \mathrm{GS} / \mathrm{s}$ and 1000000 samples were recorded for each time trace, therefore the duration of each time trace was $100 \mu \mathrm{s}$.

The second experiment was performed in an all-fiber setup, as shown in Fig. 1(b). A single mode Fabry-Perot laser from Eblana Photonics with lasing wavelength around $1.55 \mu \mathrm{m}$ was used in the experiment. At the operating temperature, the 
threshold current is $14.7 \mathrm{~mA}$. The laser was subject to optical feedback from the fiber loop. The polarization controller in the fiber loop was used to ensure polarization preserved optical feedback. The feedback round-trip time is about $42.6 \mathrm{~ns}$. The detection method for the output of the laser was the same as that in the free space experiment.

In this paper, the feedback ratio is defined as the ratio of the optical feedback power to the laser's free-running output power. The optical feedback power is measured just before it is fed back into the laser.

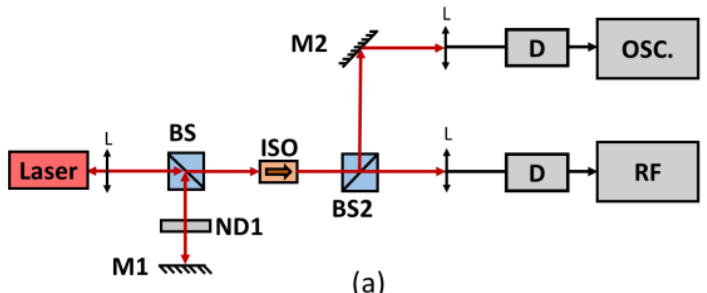

(a)

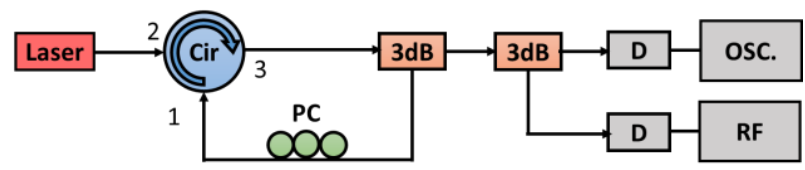

(b)

Fig. 1. (a) Free space experimental setup, (b) all-fiber experimental setup. L Lens; BS-beamsplitter; M- mirror; ND -neutral density filter; ISO - optical isolator; D-detector; OSC-oscilloscope; RF - RF spectrum analyzer; Cir-optical circulator; $3 \mathrm{~dB}$ - 3dB optical coupler; PC-polarization controller. Grey (Red in color) lines represents that the laser beam travels in free space. Black lines represents that the laser beam travels in optical fiber.

\section{ANALYSIS METHODS}

\section{A. Time delay signature}

The common methods to quantify the TD signature used are the autocorrelation (AC) function, delayed mutual information and permutation entropy [18], [20], [25], [27], [36]. The peak value of the $\mathrm{AC}$ coefficient at the feedback round trip time is used to quantify the TD signature in this paper. The autocorrelation coefficient is labelled as $\mathrm{C}$ and is defined as

$$
C(\Delta t)=\frac{<[I(t+\Delta t)-<I(t+\Delta t)>][I(t)-<I(t)>]>}{\sqrt{<[I(t+\Delta t)-<I(t+\Delta t)>]^{2}><[I(t)-<I(t)>]^{2}>}}
$$

where $\Delta t$ denotes the delay time, $I(t)$ denotes the output intensity of the laser and <.> denotes time average. The peak value at the feedback round trip time $\left(\mathrm{C}_{\mathrm{p}}\right)$ can be expressed as:

$$
C_{p}=\max |C(\Delta t)|_{\Delta t \in v_{1}\left(\tau_{d}\right)}
$$

where $\tau_{\mathrm{d}}$ is the feedback round trip time. The measured TD peak value may not be located exactly at $\tau_{\mathrm{d}}$. If a measured peak value is in the range of interval $v_{l}\left(\tau_{d}\right)=\left(\tau_{d}-\tau_{d} \times r_{l}, \tau_{d}+\tau_{d} \times r_{l}\right)$; it will be considered as the peak value at the TD. According to the experimental data, $2 \%$ is selected as the value of $r_{1}$.

\section{B. Complexity}

Several techniques have been used to quantify the complexity of chaos, such as Lyapunov exponents [37], [38], strangeness of strange attractors [39] and permutation entropy (PE) [29-30], [32],[40-41]. PE has a few advantages over other techniques, which includes easy implementation, faster computation and being robust to noise. This makes PE particularly attractive for using on experimental data, so PE is adopted to quantify the complexity of chaos in this paper.

The PE method was first introduced by Bandt et al. [40]. In this method, the measured output intensity of the laser has $\mathrm{N}$ samples $I_{t}$, where $t=1, \ldots, N$. For a given time series $\left\{I_{t}\right.$, $\mathrm{t}=1,2, \ldots, \mathrm{N}\}$, let subsets $\mathrm{S}_{\mathrm{q}}$ contain $\mathrm{M}$ samples $(\mathrm{M}>1)$ of the measured intensity and an embedding delay time $\tau=\mathrm{nT}_{\mathrm{S}}(\mathrm{n}$ is an integer number and $T_{s}$ is the reciprocal of the sampling rate), the ordinal pattern of the subset is $\mathrm{S}_{\mathrm{q}}=[\mathrm{I}(\mathrm{t}), \mathrm{I}(\mathrm{t}+\tau)$, ...I $(\mathrm{t}+(\mathrm{M}-1) \tau)] . \quad \mathrm{S}_{\mathrm{q}}$ can be rearranged as $\left[\mathrm{I}\left(\mathrm{t}+\left(\mathrm{r}_{1}-1\right) \tau\right) \leq \mathrm{I}\left(\mathrm{t}+\left(\mathrm{r}_{2}-1\right) \tau\right) \leq \ldots \leq \mathrm{I}\left(\mathrm{t}+\left(\mathrm{r}_{\mathrm{M}}-1\right) \tau\right)\right]$. Hence, any subset can be uniquely mapped into an "ordinal pattern" $\pi=\left(\mathrm{r}_{1}\right.$, $\left.r_{2}, \ldots, r_{M}\right)$, which is one of the permutations of subset $S_{q}$ with $M$ dimensions. For all the $\mathrm{M}$ ! possible permutations, the probability distribution $\mathrm{p}(\pi)$ is defined as [40]

$p()=\frac{\#\left\{t \mid t \quad N \quad\left(\begin{array}{ll}M & 1\end{array}\right) n ; S_{q} \text { has type }\right\}}{N \quad\left(\begin{array}{ll}M & 1\end{array}\right) n}$

where \# stands for "number". From the probability $\mathrm{p}(\pi)$ the permutation entropy is defined as:

$$
h(P)=-\sum p(\pi) \log p(\pi)
$$

$\mathrm{H}(\mathrm{P})$ is used to denote the normalized $\mathrm{PE}$, which can be expressed as

$$
H(P)=\frac{h(P)}{\log (M !)}
$$

The value of the normalised $\mathrm{PE}(\mathrm{H}(\mathrm{P}))$ is between 0 and $1[21]$, [29]. A value of one represents a completely stochastic process, while a value of zero indicates that the time series is completely predictable.

Bandt [40] has suggested that $M$ is chosen to be between 3 and 7 for all practical cases. $M=4$ and $M=5$ have been tested and the trend of the results agree with each other. Due to time constraints, $\mathrm{M}=4$ was chosen for this paper.

\section{EXPERIMENTAL RESULTS}

Fig. 2 shows the time traces, power spectra, autocorrelation functions and permutation entropy of the output of the laser subject to $-10.2 \mathrm{~dB}$ optical feedback at the bias current of $50 \mathrm{~mA}, 60 \mathrm{~mA}$ and $70 \mathrm{~mA}$ in the free-space experimental setup. The time series in Fig. 2(a1), (a2) and (a3) show the fluctuations of the output amplitudes, which indicates that the laser is in chaos dynamics. The second column in Fig. 2 shows the power spectra of the laser output. The results demonstrate that the bandwidth of chaos increases with increasing bias current. This is easy to understand, since the bandwidth of 
chaos is dependent on the laser's relaxation oscillation (RO) frequency and the RO frequency is proportional to the square root of the difference between the bias current and the threshold current. The third column of Fig. 2 displays the AC coefficient as a function of the delay time. In the results, the $\mathrm{AC}$ coefficients has a peak at around 5.6ns, which equals the feedback round trip time. The TD signature of chaos can be quantified by measuring the $\mathrm{AC}$ peak value at the feedback round trip time[19], [20], [22], [27]. Fig. 2(c1) shows that the $\mathrm{AC}$ peak value is 0.74 for the bias current of $50 \mathrm{~mA}$. When the bias current increases to $60 \mathrm{~mA}$, the $\mathrm{AC}$ peak value decreases to 0.72 . If the bias current is further increased to $70 \mathrm{~mA}$, the $\mathrm{AC}$ peak value decreases to 0.65 . The fourth column of Fig. 2 represents the normalized PE $(\mathrm{H}(\mathrm{p}))$ drawn against the embedding delay time. There are troughs at the feedback round trip time and its subharmonics for all three bias currents. The trough value at the feedback round time is adopted to be used to measure the complexity of chaos [32]. Fig. 2(d1) shows a deep trough at 5.6ns, where the value is 0.7 . When the bias current increases to $60 \mathrm{~mA}$, the depth of trough at delay time increases to 0.8 . Further increasing the bias current to $70 \mathrm{~mA}$, the depth of trough increases again to 0.86 .
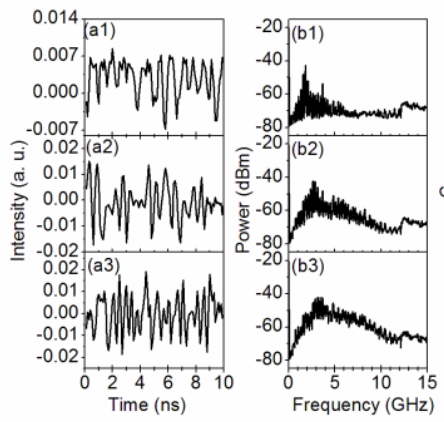
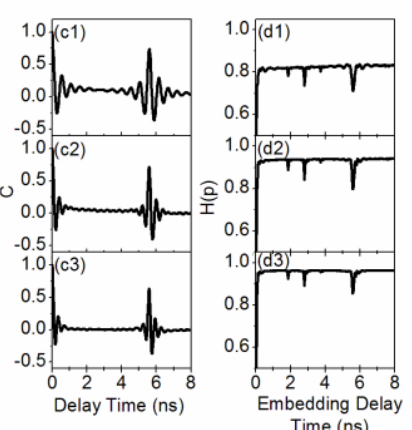

Fig. 2. The time traces (first column), RF power spectra (second column), autocorrelation coefficient curves (third column) and permutation entropy curves (fourth column)) of the chaotic signal. The top, middle and bottom rows represent bias currents of $50 \mathrm{~mA}, 60 \mathrm{~mA}$ and $70 \mathrm{~mA}$, respectively.

The results in Fig. 2 show that the values of bandwidth, TD signature and complexity of chaos with the same feedback ratio are sensitive to the bias current. The TD signature and complexity as a function of the normalized bias current with the same feedback strength used in Fig. 2 are calculated and shown in Fig. 3(a). The bias current has been normalized with the threshold current. In Fig. 3(a), a minimum TD signature has been observed at the highest bias current being measured. The complexity almost linearly increases with increasing bias current. The TD signature and complexity show an inverse relationship for most normalized bias currents except the kink at the normalized bias current between 1.55 and 1.69. The inverse relationship between the TD signature and complexity is in good agreement with the simulation result [31].

We have also performed the experiment in an all-fiber setup, as shown in Fig. 1(b). When the feedback ratio was adjusted to about $-14.3 \mathrm{~dB}$, the laser was in chaos dynamics. The TD and complexity of chaos with various bias currents were calculated and plotted in Fig. 3(b). The results show that the TD signature decreases with increasing bias current at the very beginning. When the normalized bias current reaches 1.4, a minimum TD signature is obtained. If the bias current is further increased, the TD signature will increase again. For normalized bias currents between 1.8 and 1.9, the TD signatures do not change much with the local maximum TD signature obtained. When the normalized bias current is tuned to more than 1.9, the TD signature starts to drop again. The complexity of chaos, on the other hand, initially increases with bias current until the normalized bias current reaches 1.6. After that the complexity of chaos starts to saturate at 0.93 . It is clear that the complexity has an inverse relationship with the TD signature for lower bias currents, however, the inverse relationship disappears after the normalized bias current increases beyond 1.4.
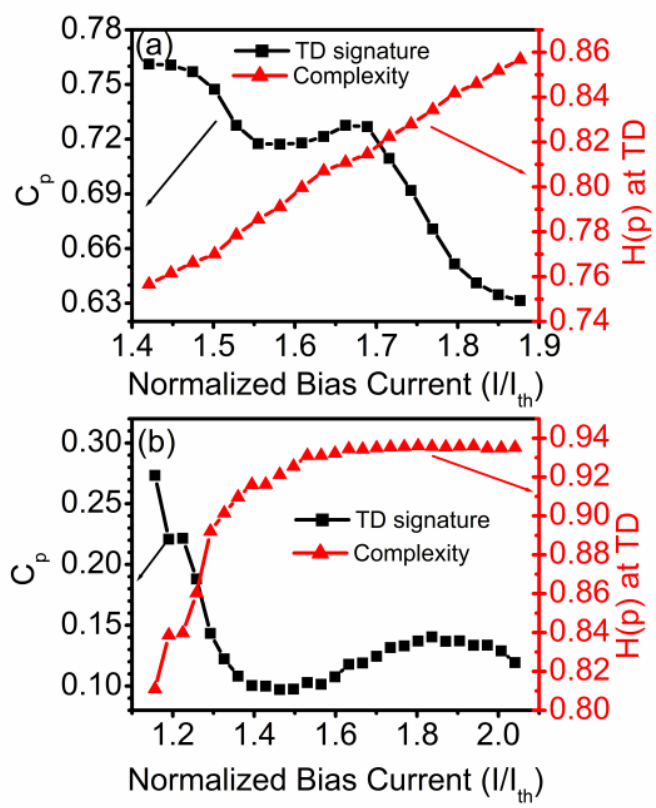

Fig. 3 The TD signature and complexity of chaos as a function of the normalized bias currents in (a) the free space experimental setup, (b) the all-fiber experimental setup.

\section{THEORETICAL MODEL AND RESULTS}

In order to understand the difference between Fig.3(a) and (b), nonlinear dynamics of semiconductor lasers with optical feedback have been numerically simulated using the Lang Kobayashi laser equations [35], as shown in Eqs (6) and (7) below.

$$
\begin{aligned}
& \frac{d E}{d t}=\frac{1}{2}(1+i \alpha)\left[G-\frac{1}{\tau_{p}}\right] E(t)+\kappa E\left(t-\tau_{\text {ext }}\right) e^{-i \omega \tau_{\text {ext }}} \\
& \frac{d N}{d t}=\frac{I}{e V}-\frac{N(t)}{\tau_{N}}-G|E(t)|^{2}
\end{aligned}
$$

In the rate equations, $\mathrm{E}(\mathrm{t})$ is the complex electric field, $\mathrm{N}(\mathrm{t})$ is the carrier number, $\alpha$ is the linewidth enhanced factor, $\kappa$ is the feedback strength, $\tau_{\mathrm{p}}$ is the photon lifetime, $\tau_{\mathrm{N}}$ is the carrier lifetime, $\tau_{\text {ext }}$ is the external cavity round-trip time, $\omega$ is the angular frequency of the laser, e is the electron charge, I is the 
laser bias current and $\mathrm{V}$ is the volume of the active region. The optical gain $\mathrm{G}$ is given by

$$
G=g_{0}\left(N-N_{0}\right) \frac{1}{1+\epsilon|E(t)|^{2}}
$$

where $\mathrm{g}_{0}$ is the differential gain, $\epsilon$ is the gain saturation factor, $\mathrm{N}_{0}$ is carrier density at transparency.

MATLAB solver dde23 was used to numerically solve Eqs. (6) and (7). Typical semiconductor laser's parameters: $\alpha=3.0$, $\tau_{\mathrm{N}}=2 \mathrm{~ns}, \tau_{\mathrm{p}}=2 \mathrm{ps}, \mathrm{g}_{0}=5 \times 10^{-12} \mathrm{~m}^{3} \mathrm{~s}^{-1}, \mathrm{~N}_{0}=1.5 \times 10^{24} \mathrm{~m}^{-3}$ and $\mathrm{V}=2.9 \times 10^{-16} \mathrm{~m}^{3}$ were chosen in the simulation. The laser's lasing wavelength was set at $860 \mathrm{~nm}$, threshold current $\mathrm{I}_{\text {th }}=37.3$ $\mathrm{mA}$ and $\tau_{\mathrm{ext}}=5.6 \mathrm{~ns}$, which corresponds to our free-space experimental setup. A temporal resolution of $\Delta \mathrm{t}=10 \mathrm{ps}$ is selected and the duration of the time series is $1 \mu \mathrm{s}$. When the feedback strength $\kappa$ is set to $60 \mathrm{~ns}^{-1}$, the laser operated at chaotic dynamics for the normalized bias current range from 1.05 to 2.0. The TD signature and complexity of chaos have been calculated and shown in Fig. 4(a). The TD signature shows an almost linear decrease with increasing bias current until the normalized bias current reaches 1.9. Further increasing the bias current, the TD signature is almost unchanged. Fig. 4(a) also shows that the complexity increases monotonically with increasing bias current until the normalized bias current reaches 1.75. Further increasing the bias current, the complexity is almost unchanged. The results in Fig. 4(a) show that the complexity is approximately in inverse relationship with the TD signature, which is qualitatively agreed with the experimental results in Fig. 3(a). However, there is no kink for the TD signature in the simulation results. Also, the complexity does not linearly increase with increasing bias current. More investigation should be done to explore the fundamental difference between experimental and theoretical results.

When the feedback strength $\kappa$ is reduced to $30 \mathrm{~ns}^{-1}$, and the other parameters kept the same as those in Fig. 4(a), the laser still operated at chaotic dynamics for the normalized bias current range from 1.05 to 2.0. Its TD signature and complexity as a function of the bias current are displayed in Fig. 4(b). The trend of the curves are different from those in Fig. 4(a). The TD signature decreases initially. When the normalized bias current reaches about 1.5 , the TD signature starts to saturate. When the normalized bias current increases to more than 1.75, The TD signature begins to increase. On the other hand, complexity increases with increasing bias current for the normalized bias current below 1.25. After that, the complexity shows saturation, and the bias current has little effect on the complexity. These results agree well with those in Fig. 3(b) except that an extra dropping of TD signature at high bias current has been observed in the experiment.

When the feedback strength $\kappa$ is further reduced to $9.32 \mathrm{~ns}^{-1}$, the dependence of the TD signature and complexity on the bias current is illustrated in Fig. 4(c). The curves are quite different compared with those in Fig. 4(a) and (b). The TD signature decreases when the normalized bias current increases from 1.05 to 1.1. After that, the TD signature starts to increase quickly with increasing bias current until the normalized bias current reaches 1.45. After that, the bias current has much less effect on the TD signature. The variation of complexity is almost inverse to that of TD signature, but the change rates for the TD and complexity vary at different bias current regions. There is a sharp increase of complexity when the normalized bias current increases from 1.9 to 1.95 . The reason for this sharp increase is due to very small fluctuation amplitude at the normalized bias current of 1.95, the laser is almost operated at steady-state.

During the experiments, we have used different wavelength lasers and different feedback round trip times to illustrate the effects. Here we have also introduced different wavelengths and feedback round trip times in the simulation. The results show that wavelength has little effect on the variation of the TD and complexity with the bias current. The feedback round trip time also has very little influence on the trend of the TD and complexity.

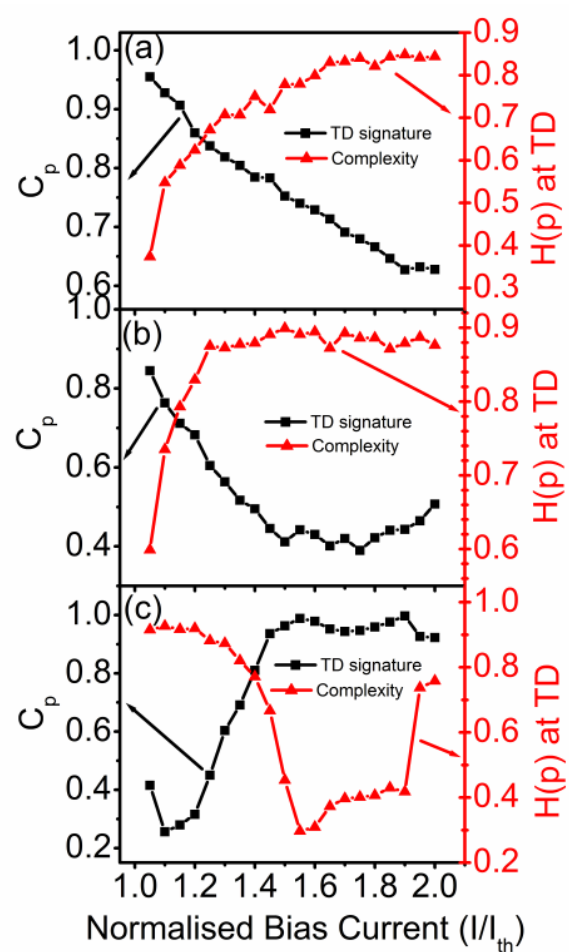

Fig. 4 Numerical results of the TD signature and complexity as a function of the normalized bias currents with a feedback strength of (a) $60 \mathrm{~ns}^{-1}$, (b) $30 \mathrm{~ns}^{-1}$, (c) $9.32 \mathrm{~ns}^{-1}$.

The maps of the TD signature and complexity with varying bias current and the feedback strength are presented in Fig.5. It can be seen in Fig. 5(a) that lower TD signature has been achieved at lower bias current with lower feedback strength. With increasing feedback strength, the lowest TD signature shifts to higher bias current. Meanwhile, the lowest TD signature decreases with decreasing feedback strength. This map is somehow different from that in Ref. [33], where lower TD signature can be achieved over a much wider range of bias currents for higher feedback ratio. This difference may be due to polarization-rotated feedback in [33], while it is polarization preserved feedback in our study.

The trend of complexity in Fig. 5(b) has some similarity with that of the TD signature in Fig. 5(a). The highest complexity is obtained at a lower bias current and a lower feedback strength. For the feedback strength between $15 \mathrm{~ns}^{-1}$ and $25 \mathrm{~ns}^{-1}$, there is an optimum bias current, where the chaos is most complex. 
This result is similar to the simulation result in [31]. This optimum bias current moves to higher bias current with an increasing feedback strength. However, for the feedback strength between $30 \mathrm{~ns}-1$ and $55 \mathrm{~ns}-1$, there is a wider bias current region where the bias current has very little effect on the complexity compared to that on the TD signature.

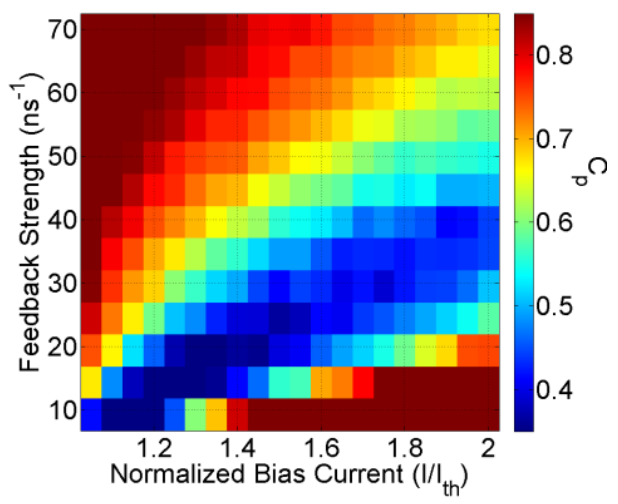

(a)

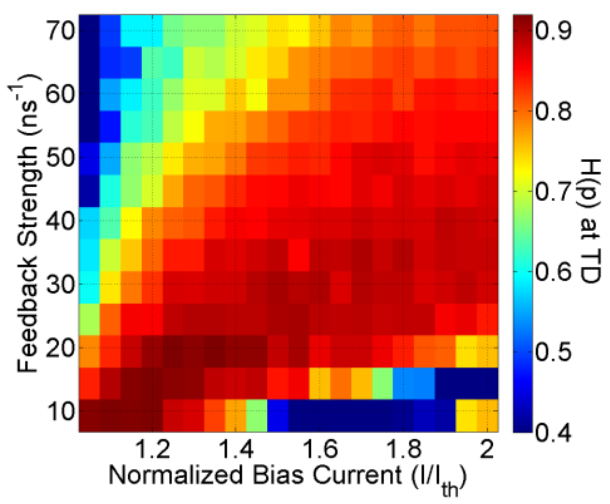

(b)

Fig. 5 Maps of (a) TD signature, (b) complexity of chaos with varying bias current and feedback strength.

\section{CONCLUSION}

The influence of bias current and the feedback strength on the complexity and time-delay signature of chaotic signals in semiconductor lasers with optical feedback has been investigated experimentally and theoretically. The time-delay signature has an approximately inverse relationship to the complexity of chaos in the free space experimental setup. However, this inverse relationship disappears at higher bias current in the all-fiber experimental setup. This disappearance is due to the decrease of feedback strength, as confirmed by the simulation results using the Lang Kobayashi laser equations. The numerical simulation results show that the TD signature has an inverse relationship with complexity for strong or low feedback strength. For intermediate feedback strength, an inverse relationship between TD signature and complexity only exists at lower bias currents, and the bias current has little effect on the complexity of chaos at higher bias currents.

\section{REFERENCES}

[1] C. Masoller, "Anticipation in the synchronization of chaotic semiconductor lasers with optical feedback," Phys. Rev. Lett., vol. 86, no. 13, pp. 2782-2785, 2001.

[2] Y. Takiguchi, K. Ohyagi, and J. Ohtsubo, "Bandwidth-enhanced chaos synchronization in strongly injection-locked semiconductor lasers with optical feedback.," Opt. Lett., vol. 28, no. 5, pp. 319-321, 2003.

[3] A. Argyris, D. Syvridis, L. Larger, V. Annovazzi-Lodi, P. Colet, I. Fischer, J. García-Ojalvo, C. R. Mirasso, L. Pesquera, and K. A. Shore, "Chaos-based communications at high bit rates using commercial fibre-optic links.," Nature, vol. 438, no. 7066, pp. 343-346, 2005.

[4] M. Sciamanna, I. Gatare, A. Locquet, and K. Panajotov, "Polarization synchronization in unidirectionally coupled vertical-cavity surface-emitting lasers with orthogonal optical injection," Phys. Rev. E, vol. 75, no. 5, p. 56213, 2007.

[5] Y. Hong, M. W. Lee, J. Paul, P. S. Spencer, and K. A. Shore, "GHz bandwidth message transmission using chaotic vertical-cavity surface-emitting lasers," J. Light. Technol., vol. 27, no. 22, 2009.

[6] J.-G. Wu, Z.-M. Wu, G.-Q. Xia, T. Deng, X.-D. Lin, X. Tang, and G.-Y. Feng, "Isochronous synchronization between chaotic semiconductor lasers over 40-km fiber links," IEEE Photonics Technol. Lett., vol. 23, no. 24, pp. 1854-1856, Dec. 2011.

[7] S. Y. Xiang, W. Pan, B. Luo, L. S. Yan, X. H. Zou, N. Jiang, N. Q. Li, and H. N. Zhu, "Message encoding/decoding using unpredictability-enhanced chaotic VCSELs," IEEE Photonics Technol. Lett., vol. 24, no. 15, pp. 1267-1269, Aug. 2012.

[8] K. E. Chlouverakis and M. J. Adams, "Optoelectronic realization of NOR logic gate using chaotic two section lasers," Electron. Lett, vol. 41, no. 6, pp. 359-360, 2005.

[9] A. Wang, N. Wang, Y. Yang, B. Wang, M. Zhang, and Y. Wang, "Precise fault location in WDM-PON by utilizing wavelength tunable chaotic laser," J. Light. Technol., vol. 30, no. 21, pp. 3420-3426, 2012.

[10]L. Xia, D. Huang, J. Xu, and D. Liu, "Simultaneous and precise fault locating in WDM-PON by the generation of optical wideband chaos," Opt. Lett., vol. 38, no. 19, p. 3762, Oct. 2013.

[11]Z. N. Wang, M. Q. Fan, L. Zhang, H. Wu, D. V. Churkin, Y. Li, X. Y. Qian, and Y. J. Rao, "Long-range and high-precision correlation optical time-domain reflectometry utilizing an all-fiber chaotic source," Opt. Express, vol. 23, no. 12, p. 15514, Jun. 2015.

[12]F.-Y. Lin and J.-M. Liu, "Chaotic lidar," IEEE J. Sel. Top. quantum Electron., vol. 10, no. 5, pp. 991-997, 2004.

[13] A. Uchida, K. Amano, M. Inoue, K. Hirano, S. Naito, H. Someya, I. Oowada, T. Kurashige, M. Shiki, S. Yoshimori, K. Yoshimura, and P. Davis, "Fast physical random bit generation with chaotic semiconductor lasers," Nat. Photonics, vol. 2, no. 12, pp. 728-732, Nov. 2008.

[14] I. Kanter, Y. Aviad, I. Reidler, E. Cohen, and M. Rosenbluh, "An optical ultrafast random bit generator," Nat Photonics, vol. 4, no. 1, pp. 58-61, Jan. 2010.

[15] A. Uchida, T. Heil, Yun Liu, P. Davis, and T. Aida, "High-frequency broad-band signal generation using a semiconductor laser with a chaotic optical injection," IEEE J. Quantum Electron., vol. 39, no. 11, pp. 14621467, Nov. 2003.

[16]D. Rontani, A. Locquet, M. Sciamanna, D. S. Citrin, and S. Ortin, "Time-delay identification in a chaotic semiconductor laser with optical feedback : a dynamical point of view," IEEE J. Quantum Electron., vol. 45, no. 7, pp. 879-891, 2009.

[17]K. E. Callan, L. Illing, Z. Gao, D. J. Gauthier, and E. Scho, "Broadband chaos generated by an optoelectronic oscillator," Phys. Rev. Lett., vol. 104, March, p. 113901, 2010.

[18]R. M. Nguimdo, P. Colet, L. Larger, and L. Pesquera, "Digital key for chaos communication performing time delay concealment," Phys. Rev. Lett., vol. 107, no. 3, p. 34103, Jul. 2011.

[19] N. Li, W. Pan, S. Xiang, L. Yan, B. Luo, X. Zou, L. Zhang, and P. Mu, "Photonic generation of wideband time-delay- signature-eliminated chaotic signals utilizing an optically injected semiconductor laser," IEEE J. Quantum Electron, vol. 48, no. 10, pp. 1339-1345, 2012.

[20] J.-G. Wu, Z.-M. Wu, G.-Q. Xia, and G.-Y. Feng, "Evolution of time delay signature of chaos generated in a mutually delay-coupled semiconductor lasers system," Opt. Express, vol. 20, no. 2, p. 1741, Jan. 2012.

[21] S. Y. Xiang, W. Pan, B. Luo, L. S. Yan, X. H. Zou, N. Li, and H. N. Zhu, "Wideband Unpredictability-enhanced chaotic semiconductor lasers with dual-chaotic optical injections," IEEE J. Quantum Electron, vol. 48, no. 8, pp. 1069-1076, 2012. 
[22] Y. Hong, "Experimental study of time-delay signature of chaos in mutually coupled vertical-cavity surface-emitting lasers subject to polarization optical injection.," Opt. Express, vol. 21, no. 15, pp. 17894-17903, 2013.

[23] A. Wang, Y. Wang, Y. Yang, M. Zhang, H. Xu, and B. Wang, "Generation of flat-spectrum wideband chaos by fiber ring resonator," Appl. Phys. Lett., vol. 102, no. 3, p. 31112, 2013.

[24]Z.-Q. Zhong, Z.-M. Wu, J.-G. Wu, and G.-Q. Xia, "Time-delay signature suppression of polarization-resolved chaos outputs from two mutually coupled vcsels," IEEE Photonics J., vol. 5, no. 2, p. 1500409, Apr. 2013.

[25] Y. Hong, P. S. Spencer, and K. A. Shore, "Wideband chaos with time-delay concealment in vertical-cavity surface-emitting lasers with optical feedback and injection," IEEE J. Quantum Electron., vol. 50, no. 4, pp. 236-242, 2014.

[26] Y. Hong, "Flat broadband chaos in mutually coupled vertical-cavity surface-emitting lasers," IEEE J. Sel. Top. Quantum Electron., vol. 21, no. 6, p. 1801007, 2015

[27] S.-S. Li and S.-C. Chan, "Chaotic time-delay signature suppression in a semiconductor laser with frequency-detuned grating feedback," IEEE $J$. Sel. Top. Quantum Electron., vol. 21, no. 6, p. 1800812, 2015.

[28] A. Wang, B. Wang, L. Li, Y. Wang, and K. A. Shore, "Optical heterodyne generation of high-dimensional and broadband white chaos," IEEE J. Sel. Top. Quantum Electron., vol. 21, no. 6, p. 1800710, 2015.

[29] J. P. Toomey and D. M. Kane, "Mapping the dynamic complexity of a semiconductor laser with optical feedback using permutation entropy," Opt. Express, vol. 22, no. 2, p. 1713, Jan. 2014.

[30] N. Li, W. Pan, A. Locquet, and D. S. Citrin, "Time-delay concealment and complexity enhancement of an external-cavity laser through optical injection.," Opt. Lett., vol. 40, no. 19, pp. 4416-4419, 2015.

[31]K. Kanno, A. Uchida, and M. Bunsen, "Complexity and bandwidth enhancement in unidirectionally coupled semiconductor lasers with time-delayed optical feedback," Phys. Rev. E, vol. 93, no. 3, p. 32206, Mar. 2016.

[32]D. Rontani, E. Mercier, D. Wolfersberger, and M. Sciamanna, "Enhanced complexity of optical chaos in a laser diode with phase-conjugate feedback," Opt. Lett., vol. 41, no. 20, p. 4637, Oct. 2016.

[33] N. Oliver, M. C. Soriano, D. W. Sukow, and I. Fischer, "Dynamics of a semiconductor laser with polarization-rotated feedback and its utilization for random bit generation," Opt. Lett., vol. 36, no. 23, pp. 4632-4634, 2011.

[34] A. Quirce, A. Valle, H. Thienpont, and K. Panajotov, "Enhancement of chaos bandwidth in VCSELs induced by simultaneous orthogonal optical injection and optical feedback," IEEE J. Quantum Electron., vol. 52, no. 99 , p. $2400609,2016$.

[35]R. Lang and K. Kobayashi, "External optical feedback effects on semiconductor injection laser properties," IEEE J. Quantum Electron., vol. 16, no. 3, pp. 347-355, Mar. 1980.

[36]C.-H. Cheng, Y.-C. Chen, and F.-Y. Lin, "Chaos time delay signature suppression and bandwidth enhancement by electrical heterodyning," Opt. Express, vol. 23, no. 3, p. 2308, Feb. 2015.

[37]M. T. Rosenstein, J. J. Collins, and C. J. Deluca, "A practical method for calculating largest Lyapunov exponents from small data sets," Phys. D, vol. 65 , no. 1-2, pp. 117-134, 1993.
[38]H. Kantz, "A robust method to estimate the maximal Lyapunov exponent of a time series," Phys. Lett. A, vol. 185, no. 1, pp. 77-87, 1994.

[39]P. Grassberger and I. Procaccia, "measuring the strangeness of strange attractors," Phys. D Nonlinear Phenom., vol. 9, pp. 189-208, 1983.

[40]C. Bandt and B. Pompe, "Permutation entropy: a natural complexity measure for time series," Phys. Rev. Lett., vol. 88, no. 17, p. 174102, Apr. 2002.

[41] L. Zunino, M. C. Soriano, I. Fischer, O. A. Rosso, and C. R. Mirasso, "Permutation-information-theory approach to unveil delay dynamics from time-series analysis," Phys. Rev. E, vol. 82, p. 046212, 2010.

Songkun Ji received the B.A. degree in physics in State University of New York at Geneseo in 2013 and the M.Sc. degree in Electronic Engineering in Bangor University in 2015. $\mathrm{He}$ is currently working toward the Ph.D. degree in Electronic Engineering.

His current research interests are in the areas of nonlinear dynamics of semiconductor lasers subject to optical injection and optical feedback and photonic microwave generation

Yanhua Hong received the B.Sc. degree in physics from Fujian Normal University, Fuzhou, China, the M.S. degree in physics from Beijing Normal University, Beijing, China, and the Ph.D. degree in optics from the Institute of Physics, Chinese Academy of Sciences, Beijing, in 1987, 1990, and 1993, respectively.

She was a Lecturer with Beihang University, Beijing, from 1993 to 1997. Since 1997, she has been with Bangor University, Bangor, U.K., where she became a Permanent Research Staff in 2007, Lecturer in 2013 and Senior Lecturer in 2016. She is the author or co-author of more than 170 journal and conference papers. Her current research interests include nonlinear dynamics in edge-emitting semiconductor lasers, vertical-cavity surface-emitting lasers, semiconductor optical amplifiers, and dynamic networking for future optical networks.

Dr. Hong is a member of IEEE and a senior member of the Optical Society of America 